\title{
Closed Loop Performance Analysis of Classical PID and Robust H-infinity Controller for VTOL Unmanned Quad Tiltrotor Aerial Vehicle
}

\author{
Navya Thirumaleshwar Hegde ${ }^{1}$, Aldrin Claytus Vaz ${ }^{2}$, C. G. Nayak ${ }^{2 *}$ \\ ${ }^{1}$ Department of Aeronautical and Automobile Engineering, Manipal Institute of Technology, \\ Manipal Academy of Higher Education, Manipal, India, 576104 \\ navya.hegde@manipal.edu \\ ${ }^{2}$ Department of Instrumentation and Control Engineering, Manipal Institute of Technology, \\ Manipal Academy of Higher Education, Manipal, India, 576104 \\ aldrin.claytus@gmail.com,cg.nayak@manipal.edu* \\ *Corresponding Author: cg.nayak@manipal.edu
}

Received: March 5, 2021. Revised: September 11, 2021. Accepted: September 30, 2021. Published: October 4, 2021.

\begin{abstract}
Unmanned Aerial Vehicles (UAVs) guidance, control and navigation have directed the attention of many researchers in both aerospace engineering as well as control theory. Due to the unique rotor structure of Tiltrotor hybrid UAVs, they exhibit special application value. Quad Tiltrotor UAVs set up a distinctive platform that satisfies the needs of the varying mission requirements by combining the conventional features of high-speed cruise capabilities of an aircraft and hovering capabilities of a helicopter and by tilting its four rotors. The aim of this research article is to control the attitude and altitude of the UAV in the presence of uncertainty using two different control techniques. This paper addresses the comparative analysis of the robust $\mathrm{H}$-infinity controller with classical PID control designs for the transition manoeuvre of a hybrid UAV: the VTOL Tiltrotor UAV. The proposed controllers achieve hover to cruise mode transition and vice-versa. The main idea behind the design of controller is to model and analyze the UAV's position and attitude dynamics. The desired flight trajectory and the transition manoeuvre is achieved by controlling the tilt angle in $15^{\circ}$ intervals from $90^{\circ}$ to $0^{\circ}$ and vice-versa. Performance index subjected to IAE is estimated and compared for both the controllers in the presence of noise, disturbances and uncertainties. The results of simulation illustrate that the robust $H$-infinity controller achieves better transition, good adaptability, robust performance and robust stability for the whole flight envelope when compared with the PID controller.
\end{abstract}

Keywords-Aerospace Control, Altitude control, Attitude control, H-infinity Control, Quad VTOL Tiltrotor UAV, Transition Flight Modeling, Unmanned Aerial vehicles.

\section{INTRODUCTION}

U NMANNED aerial vehicles (UAVs) refers to the airvehicles with no pilots onboard and are generally termed as 'drones' [1] which can perform tasks that are much expensive to be carried out by pilot operated vehicles or dangerous for human beings. Due to the diverse range of applications exhibited by the UAVs, they are extensively gaining research interest in recent years. UAVs find applications in industrial, military and civil such as weather research, pipeline inspection, search and rescue, surveillance, intelligence and reconnaissance missions at higher altitude, natural disaster risk management and environmental observation. UAVs can be broadly classified into fixed-wing, rotary-wing, flapping-wing, blimps, and convertible/hybrid configurations. Each of the mentioned types has their own advantages and disadvantages depending on the mission goal and on the characteristics of the environment in which they are operated. The Tiltrotor fit into the hybrid/convertible configuration UAV, which combines the advantages of Vertical Take-off and Landing (VTOL) capabilities of rotary wing and horizontal flight of fixed wing.

The Tiltrotor crafts has the major advantage of elimination of runway, efficient endurance and high forward velocity. During the VTOL phase, the axes of the rotors will be perpendicular to the ground and remain parallel during the cruise phase. By tilting the rotors, the transition between horizontal and vertical flight and vice versa is achieved [2]. Additional benefits of rotorcrafts include high speed at less tilt angle, omni-directional and flying stationary in hover. The motors are situated on the wings tip of the Tiltrotors, the nacelles and poles turn the rotors instead of entire wing or vehicle, resulting in lower weight and less power consumption. Compared to other hybrid UAVs, the Tiltrotors are researched proficiently due to its controllability and stability in vertical flight [3]. 
An UAV is a complex physical system consisting limited information such as aerodynamic coefficients and variation in sensor noise. The popular classical Proportional-IntegralDerivative (PID) controller is a method to control numerous system dynamics [3]. PID can also be used in UAV flight control systems similar to their application in industries. The key selling point of PID implementation is its simple architecture, easily tunable, and also having a wide range of performance. Although, the PID controller is simple to implement, the computation of controller gains through manual tuning is a time-consuming and complex task which requires caution and experience. Moreover, from the UAV system designer point of view, the PID tuning is tedious and ambiguous, so trial and error methods were used for tuning [4], [5].

The vehicle/system to be controlled, actuators and sensors, and a controller are the key components of a feedback control system. The main motivation and aim to design a feedback control system/robust controller is to minimize the disturbances, uncertainties (unstructured or structured), model inaccuracies and noise. During the flight controller design, $\mathrm{H}$ infinity controller is used in the presence of external Dryden turbulence to accomplish both stability and performance [6].

The unique arrangement of the rotor structure of Tiltrotor hybrid UAVs finds their use in special applications. The various levels of success achieved in controlling the UAVs using different control methods are discussed in literatures. A fully autonomous FCS for a UAV is needed to accomplish various tasks [7]. To track trajectory, position and orientation, modern control systems from classical linear/nonlinear controller to fuzzy/neural network intelligent controllers have been mostly developed for fixed/rotary-wing type UAVs.

A PID controller is proposed for a novel dual Tiltrotor aircraft for VTOL flight mode and good control effect is observed in the results [8]. The authors of [9] proposed a Quadrotor where, in the horizontal mode the rotors are oriented backward and in the VTOL mode they are configured downward and proved to be more robust to wind disturbances.

Authors in [10] implemented a control algorithm for the transition manoeuvre of a Quad Tiltrotor UAV on hardware. A novel $\mu$-synthesis based robust $\mathrm{H}$-infinity controller for a fixed-wing UAV is proposed for linearized lateral directional and longitudinal dynamics [11]. From the closed-loop frequency responses, controller performances, nominal and perturbed system responses, it can be deduced the proposed $\mu$ synthesis method is a good solution for controlling UAV dynamics under the aforementioned conditions. Real-time HIL (Hardware-In-Loop) simulations were conducted by authors of [12] by implementing a novel optimized robust controller.

In [13], [14] rotational attitude moments are regulated by implementing a non-linear robust H-infinity PID controller. Authors in [15], [16] proposed a mixed hybrid PID and Hinfinity controller against wind disturbances. The methods presented in the literature, mostly discusses on the dynamic non-linear model control of UAVs with simple trajectories in the presence of uncertainties. The studies involving measurement noise, payload variation, sinusoidal and impulse wind disturbances, couldn't perform well in executing specified tasks. So far in the literature, robust controller for VTOL Tiltrotor has not been considered much in performing good stability. In the transition mode, the adaptability of the controller can be improved using an active modeling technique. The emphasis should be on the design of robust controller which overcomes the range of stability and the dynamic uncertainties of non-linear systems. Research must be focused on improving UAV autonomy by employing numerous control methods in varying environments [17], [18].

In [19] the design and implementation of the pitch and roll autopilots for a Tactical Unmanned Aerial Vehicle (TUAV), developed by Middle East Technical University (METU). Author combined the development of the classical PID and robust $\mathrm{H}$-infinity controllers for the pitch and roll autopilots in MATLAB/Simulink environment. The results of the simulation illustrate the performances of the designed in presence of uncertainties and sensor noises.

The development of practical coupling rejection control scheme based on wind tunnel results to an unconventional flight platform problem: a high lift-to-drag ratio TTR flying wing UAV is presented in [20]. In [21], a distributed LeaderFollower control method is presented based on neighbor trajectory generation topology, where the trajectory of each $\mathrm{UAV}$ in the formation is generated online considering of the reference trajectory given to the leader. A model-driven control design based on the specialization of Model- Based Systems Engineering approach combined with the real-time UML/MARTE, hybrid automata and the Extended Kalman Filter (EKF) algorithm for quadrotor UAVs is designed in [22].

An overview of VTOL Tiltrotor UAV in terms of working principle and a brief discussion of various control configurations in three different flight modes are presented [18] and discusses the framework of mathematical modeling and different control schemes in line with UAV.

A 6DOF mathematical model of an Autonomous VTOL Tiltrotor UAV is derived using Newton-Euler's EOM/first principle theory for take-off, transition and cruise flight modes. As the developed UAV model is non-linear, the system is linearized around the trim points using Taylor series expansion/small perturbation theory [23].

The design of an autonomous flight control system for the VTOL Quad Tiltrotor UAV using a robust H-infinity controller capable of stabilizing the UAV in-flight during the mode of transition and high manoeuvrability is dealt in [23].

A Quad Tiltrotor UAV dynamic model based on NewtonEuler formulation is mathematically developed for vertical, transition, and horizontal flight phases in [18]. The proposed robust $\mathrm{H}$-infinity controller is evaluated and analyzed via simulation to control different flight dynamic modes of the UAV in [23]. The results of the simulation show that the Tiltrotor UAV achieves transition successfully with disturbances, noise and uncertainties being present.

The main contribution of this research work is to compare and improve the disturbance rejection properties of the proposed VTOL Quad Tiltrotor hybrid UAV, by designing 
two different control algorithms i.e., PID and H-infinity, for position and orientation trajectory regulation.

The organization of the rest of the article is, Section II gives an overview of the flight control systems (PID and $\mathrm{H}$-infinity) designed for the proposed UAV is discussed along with the results, discussions, achievements and limitations. This is followed by the comparison of classical PID and robust Hinfinity controller performances through simulation in Section III Finally, Section IV concludes the paper.

\section{FoRMULATION OF ClASSICAL PID CONTROL DESIGN FOR VTOL QUAD TILTROTOR UAV}

The altitude and attitude of the UAV is controlled by the control input ' $u$ ' w.r.t. the reference input is given by (1)

$$
u(t)=K_{p} e(t)+K_{i} \int e(t) d t+K_{d} \frac{d}{d t} e(t)
$$

Where error can be formulated as in (2)

$$
e(t)=s_{p}-p_{v}(t)
$$

Here ' $p_{v}(t)$ ' is the control variable at instantaneous time at ' $s_{p}$ ', is the reference position.

To establish a desired position, high quality controller must make yaw, pitch and roll angles to stay stable and constant.

The following assumptions are used in PID design methodology along with Pythagoras theorem:

- The UAV is a rigid body with symmetrical structure and constant mass.

- The CG and center of mass coincides.

- The Inertia matrix $(I)$ is negligible.

- Thrust is proportional to the square of the propellers speed.

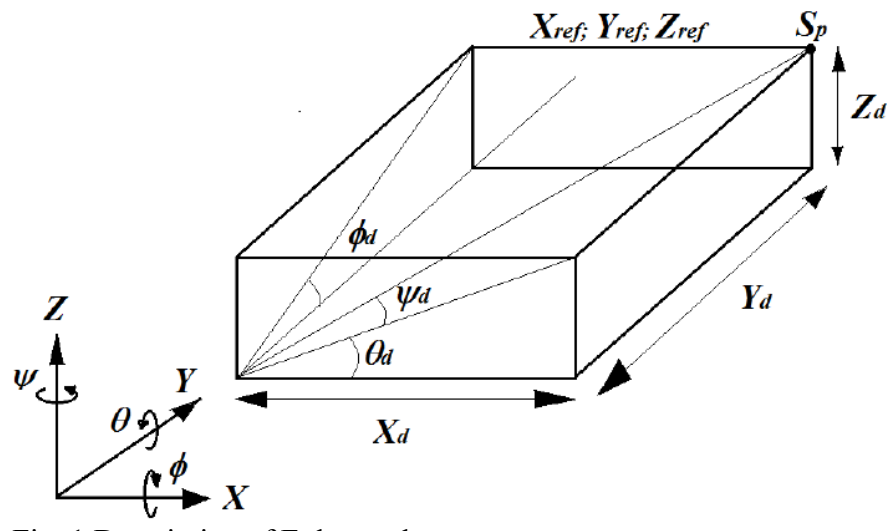

Fig. 1 Description of Euler angles

The whole methodology for the design of controller is shown in Fig. 1 based on the above-mentioned assumptions where their rotational angles can be identified using a desired position. The roll, pitch and yaw angles are depicted in (3),

$$
\begin{gathered}
\phi_{d}=\tan ^{-1}\left(Z_{d} / Y_{d}\right) \\
\theta_{d}=\sin ^{-1}\left(X_{d} / \sqrt{Z_{d}^{2}+X_{d}^{2}}\right) \\
\phi_{d}=\cos ^{-1}\left(Y_{d} / \sqrt{X_{d}^{2}+Y_{d}^{2}+Z_{d}^{2}}\right)
\end{gathered}
$$

The roll, pitch, and yaw angles during the UAV motion is shown in Fig. 1. Better position is achieved by very good tracking of the desired angles. Every instantaneous position in the inertial frame creates a cube with the changing dimension and recalculates the rotational angles, by considering the critical value of pitch angle $\theta<\frac{\pi}{2}$.

The input needed to control the spatial location $(X, Y, Z)$ of the designed geometric model are given in (4),

$$
\begin{aligned}
& u_{X}=K_{p}\left(X_{d}-X\right)+K_{i} \int_{0}^{t}\left(X_{d}-X\right)+K_{d} \frac{d}{d t}\left(X_{d}-X\right) \\
& u_{Y}=K_{p}\left(Y_{d}-Y\right)+K_{i} \int_{0}^{t}\left(Y_{d}-Y\right)+K_{d} \frac{d}{d t}\left(Y_{d}-Y\right) \\
& u_{Z}=K_{p}\left(Z_{d}-Z\right)+K_{i} \int_{0}^{t}\left(Z_{d}-Z\right)+K_{d} \frac{d}{d t}\left(Z_{d}-Z\right)
\end{aligned}
$$

Where $K_{p}, K_{i}$ and $K_{d}$ are PID gains for position control.

The desired angles are represented in (5)

$$
\begin{aligned}
& u_{\phi}=K_{p a}\left(\phi_{d}-\phi\right)+K_{i a} \int_{0}^{t}\left(\phi_{d}-\phi\right)+K_{d a} \frac{d}{d t}\left(\phi_{d}-\phi\right) \\
& u_{\theta}=K_{p a}\left(\theta_{d}-\theta\right)+K_{i a} \int_{0}^{t}\left(\theta_{d}-\theta\right)+K_{d a} \frac{d}{d t}\left(\theta_{d}-\theta\right) \\
& u_{\psi}=K_{p a}\left(\psi_{d}-\psi\right)+K_{i a} \int_{0}^{t}\left(\psi_{d}-\psi\right)+K_{d a} \frac{d}{d t}\left(\psi_{d}-\psi\right)
\end{aligned}
$$

Where $K_{p a}, K_{i a} \$$ and $K_{d a}$ are PID gains for desired rotation angles.

Multiple PID controllers for Tiltrotor UAVs have been implemented successfully for different flight modes [17]. The transition is achieved via mode switching algorithms by applying smooth control commands. SISO PID controllers that includes three sequential loop closure techniques are developed for a MIMO VTOL fixed-wing UAV [23].

The gains of the proposed PID control algorithm to control the attitude, altitude and velocity control outputs of the UAV are tuned through Ziegler-Nichols approach [10], [24]. The stability and performance of the proposed PID algorithm is verified and analyzed through simulations [23].

The complete flight envelope is simulated on the 6DOF Quad VTOL Tiltrotor UAV mathematical model in MATLAB/SIMULINK for 12 secs. The altitude and the position controllers will generate the required thrust for the reference altitude and desired xyz coordinates, respectively. The UAV starts to function when the velocity and the thrust signals operates the motors. The flowchart and the block diagram of the control algorithm of the entire flight simulation is referred from [4], [23].

\section{A. Results and Discussions with PID Controller}

The operation of the UAV begins from rest under the influence of ground effect. The vertical take-off mode starts after few instants of hover. This mode is achieved by generating the thrust from all the four rotors to maintain a particular height by overcoming its weight out from the ground effect. The change in altitude and attitudes during flight simulation is noted. The altitude is increased by decreasing the pitch angle gradually from $90^{\circ}$. The gears tilts the four proprotors synchronously to pitch the UAV towards horizontal by increasing the horizontal speed [4], [23]. 
Tilt Angle in Hover-Transition-Cruise-Back Transition Phase

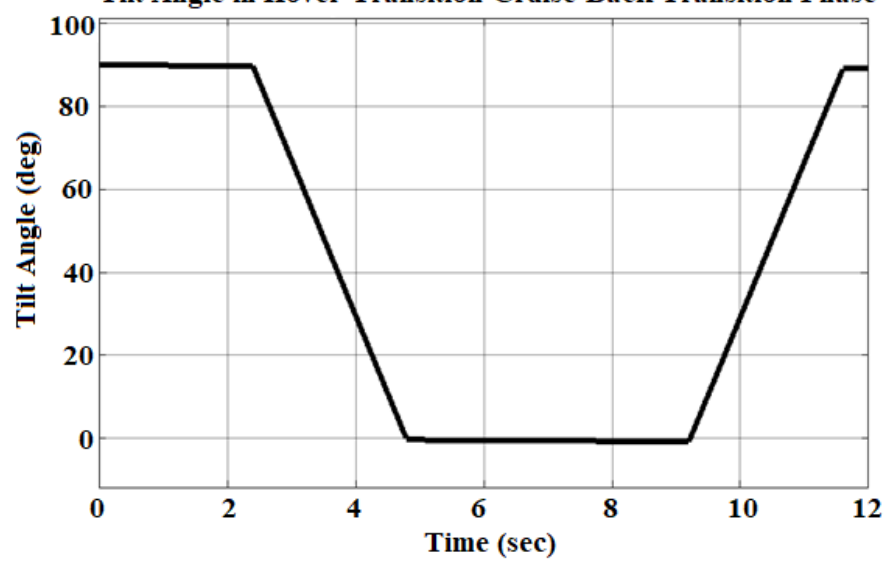

Fig. 2 Tilt angle change during entire flight scenario

The differential thrusts of the right and left motors regulates the roll dynamics, the yaw dynamics is regulated by the counteractive moments generated by the rotation of the rotors, the differential thrust generated by the front and the rear rotors regulates the pitch dynamics, during the transition phase. The tilt angle reaches to $0^{\circ}$ when the maximum reference altitude is reached and the UAV flies in horizontal mode as shown in Fig. 2 [4].

The change in attitude and altitude and also the change in their errors are shown in Fig. 3 \& Fig. 4. The graph infers that there is no overshoot in the system response and the steady state error is driven to zero.

In the hover, altitude is maintained at $5 \mathrm{~m}$ for several seconds. The altitude is then increased to about $10 \mathrm{~m}$ to achieve the transition. After the transition, horizontal flight is performed at $14 \mathrm{~m}$ altitude and the flight glides at around $12 m-14 m$ altitude before back-transition takes place. Then, at an altitude of $12 \mathrm{~m}$, the back-transition flight controller is activated. The tilt angle gradually increases to $90^{\circ}$ with the decrease in the flight speed of the UAV. At the last, the hover control system is activated after the completion of backtransition and the flight will be landed as shown in Fig. 3.
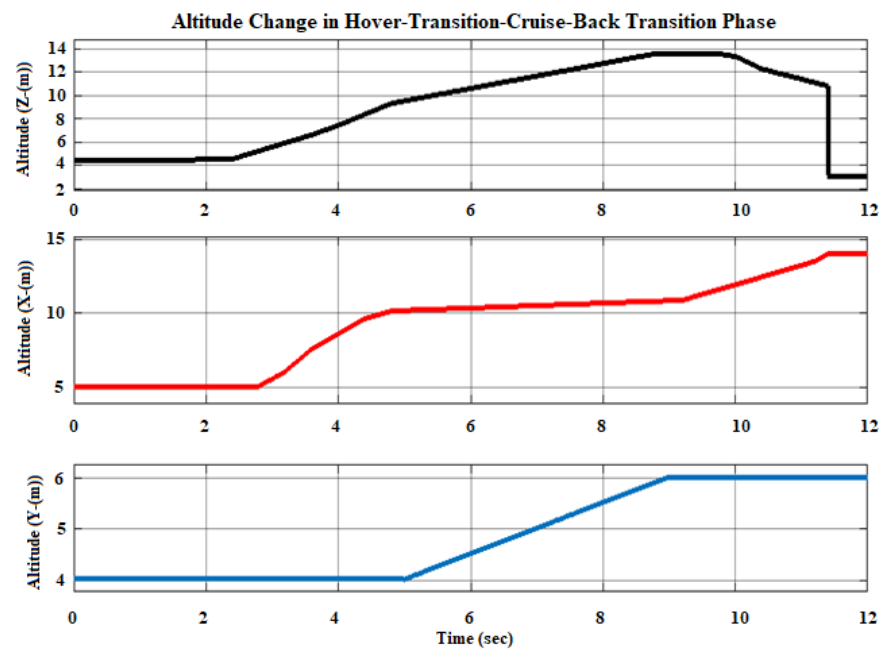

(a)
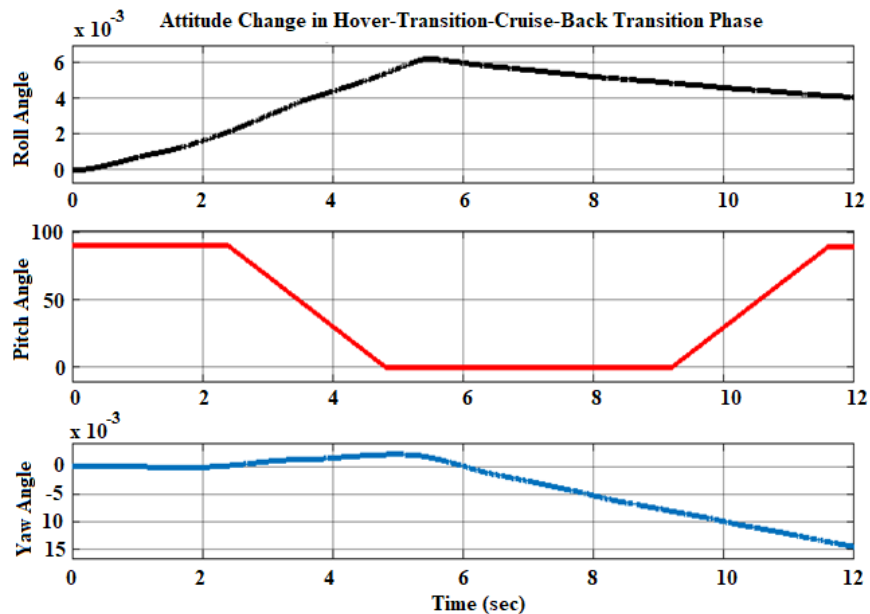

(b)

Fig. 3 (a) Altitude, (b) Attitude. The change in altitude and attitude during entire flight

The most challenging part of a VTOL flight simulation of a UAV is achieving horizontal to vertical transition and viceversa. This is achieved by gradually changing the tilt angle of the rotor in steps of $15^{\circ}$ interval. There will be different value of thrust, velocity, lift and pitching moment at each tilt angle, as shown in Fig. 5. The path traced by the UAV during its entire flight is shown in Fig. 6 [4].
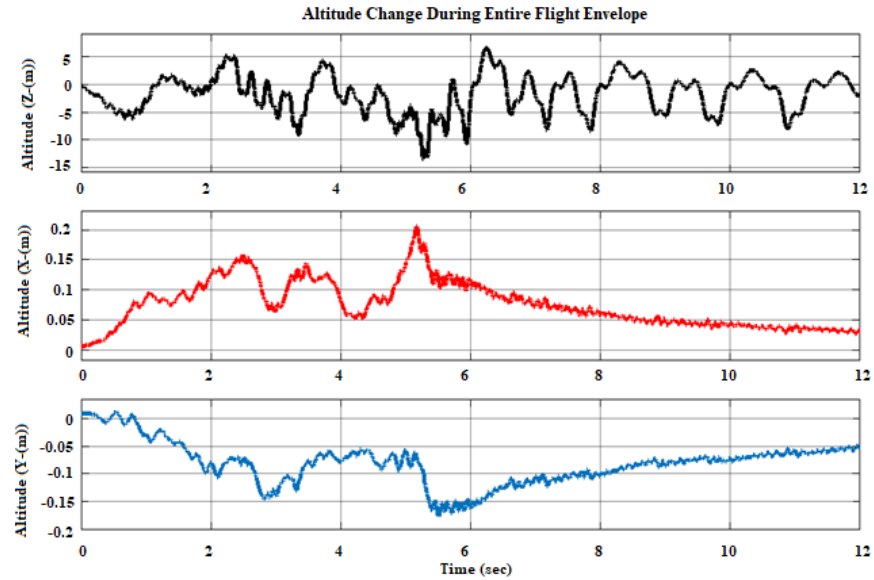

(a)
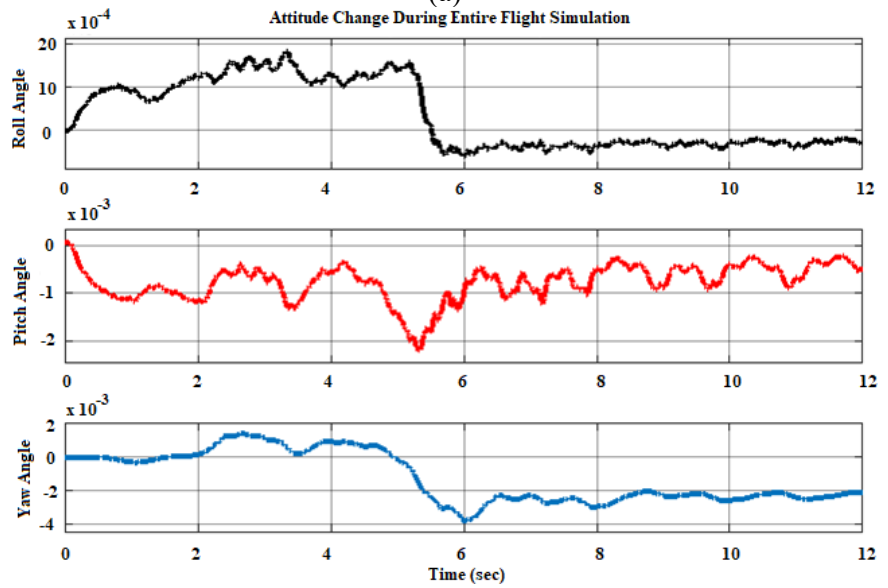

(b)

Fig. 4 (a) Altitude, (b) Attitude. The change in altitude and attitude error during entire flight. 


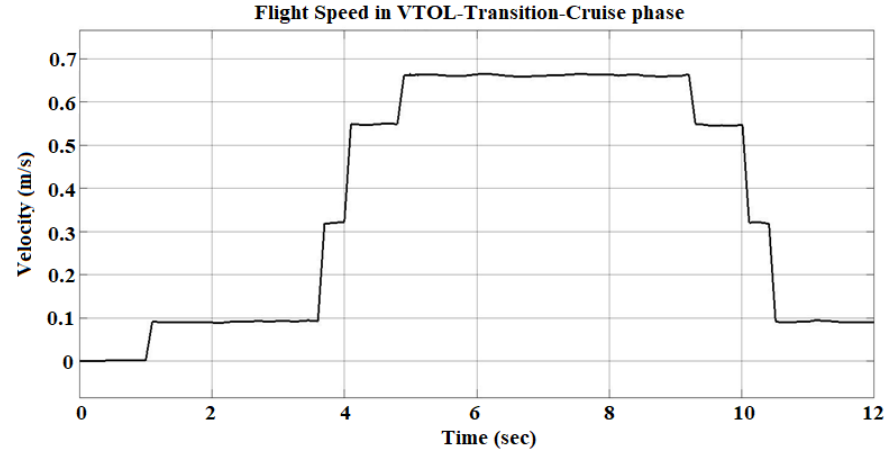

Fig. 5 Flight speed during entire flight

Fig. 6 shows the system response with PID controller when atmospheric turbulence acting on the model is zero. The PID control has achieved reference trajectory in the given time. Hence, the PID controller works well in the absence of disturbances and uncertainties. In Fig. 7 and Fig. 8, the PID control effort with the addition of atmospheric turbulence is plotted. The designed PID controller for the UAV in presence of air gust is getting disturbed from planned trajectory. The turbulence effect chosen for this paper is Dryden Wind Turbulence Model. This model is represented by the Dryden velocity spectra (is a stochastic process) to incorporate turbulence effects to the UAV model by using band-limited white noise. The trajectory traced by the UAV during entire flight simulation with turbulence acting on the system is shown in the Fig. 8.

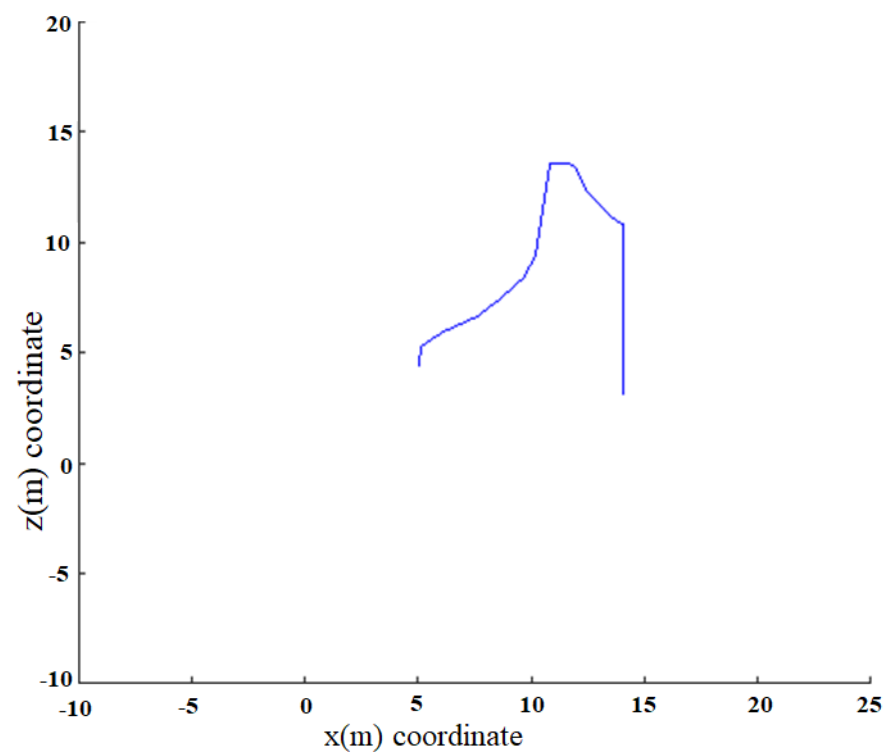

Fig. 6 The flight path of Quad Tiltrotor UAV

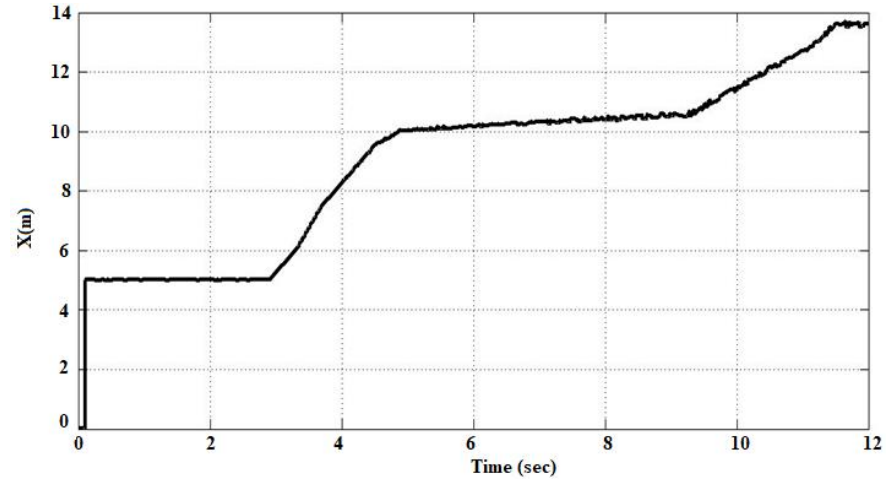

(a)

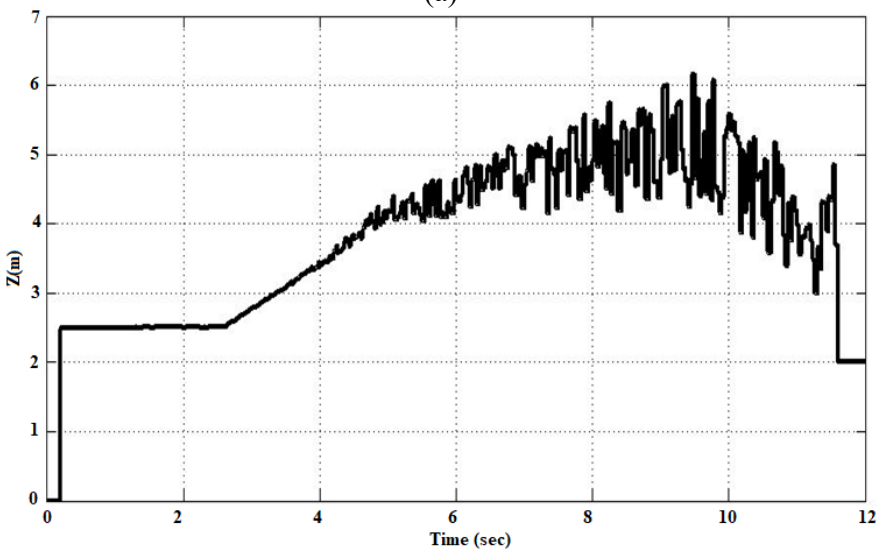

(b)

Fig. 7 (a) X(m), (b) $Z(m)$. The Change Altitude $x(m)$ and $z(m)$ in the presence of disturbance during entire flight scenario.

The Fig. 7 depicts the change in flight speed and altitude $(z(m))$ during entire simulation with Dryden Wind Turbulence effects. Graph infers, due to the presence of random noise and disturbance (air gust), PID controller fails to track the reference and has maximum overshoot. Fig. 7 \& Fig. 8 shows that the UAV becomes more unstable and not able to fly stable for the given environmental conditions. Responses are highly oscillating, and UAV is missing the trajectory. The problem faced by the unstable UAV in presence of disturbance, model inaccuracies, noise and uncertainties is overcome by designing a robust $\mathrm{H}$-infinity controller [4], [23], [25].

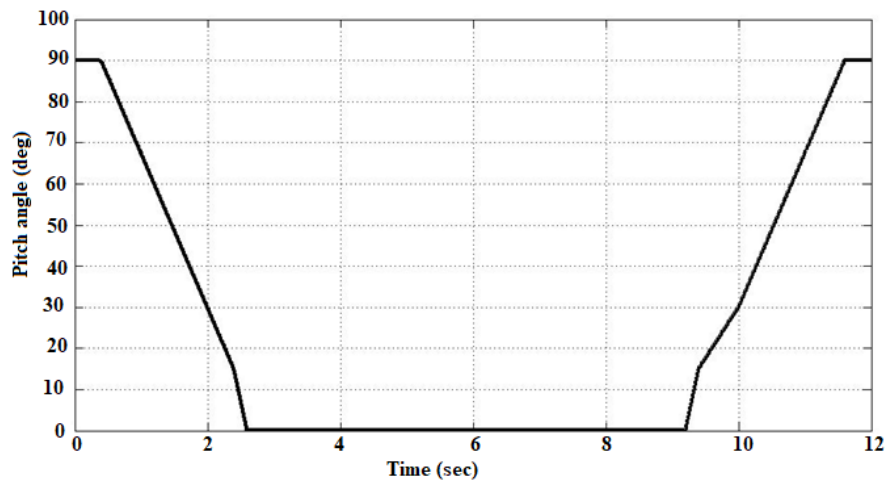

(a) 


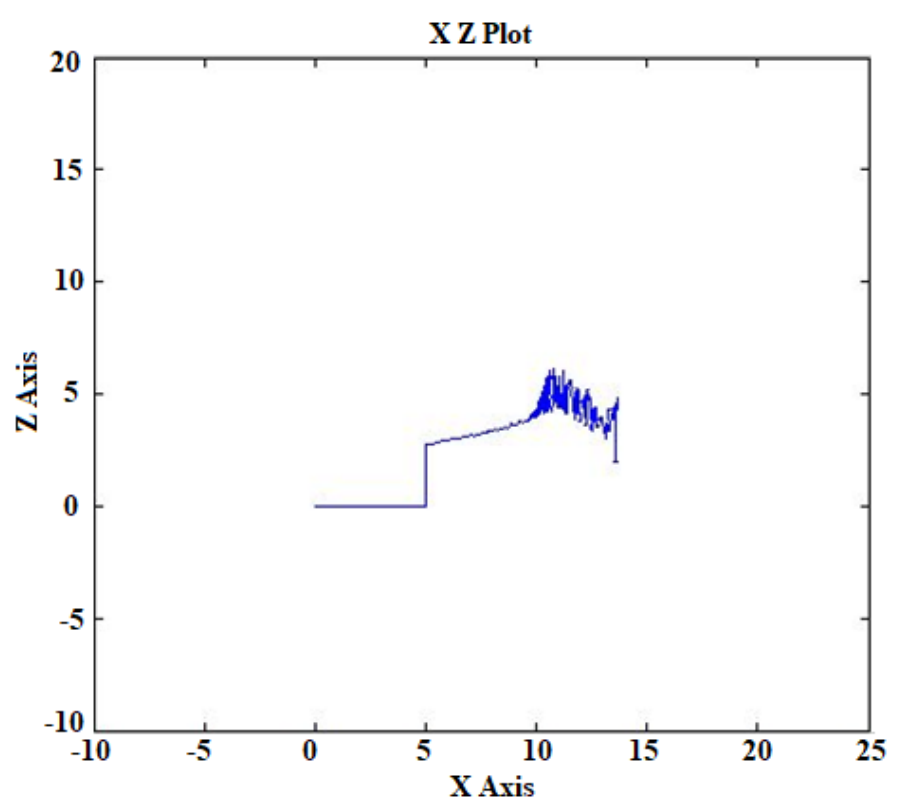

(b)

Fig. 8 (a) Pitch angle, (b) 2D flight path. Tilt Angle change and 2D flight path UAV during entire flight scenario.

\section{PERFormance ANALYSIS OF ClASSICAL PID AND RoBUST H-INFINITY CONTROLLER}

The VTOL Tiltrotor UAV flight control system is divided into three modes: forward, VTOL and transition flight mode. For the altitude and attitude control of the UAV, three PID or $\mathrm{H}$-infinity controllers are used in each flight mode for the roll, pitch and yaw control [19].

The performance analysis of the designed controller using classical control theory and the robust control theory has been carried out. Comparative analysis of both the controllers performance is simulated in MATLAB/SIMULINK for an unit step input. Simulations are carried out on a nominal model of the UAV, by considering uncertainties, Dryden turbulence effects and noise in the system's model.

During the evolution of a UAV control system, the real vehicle dynamics are approximated using mathematical model. The vehicle's stability and performance will be effected by the model uncertainty. The robust control theory optimizes performance without sacrificing stability in the presence of uncertainty and reduces cost and the design efforts required.

The performance of the robust $\mathrm{H}$-infinity controller is measured w.r.t. the peak singular value/gain of a given system. The system performance will improve as the gain starts decreasing. Robustness analysis of the system is to find out the maximum quantity of uncertainty compatible with a given performance level and stability. The robust stability margin (robstab function) is the maximum quantity of uncertainty that the system can tolerate being stable system. The robust performance margin (robgain function) is the maximum amount of uncertainty the system can tolerate while having a peak gain less than $\gamma$.

The classical PID and robust H-infinity control algorithms for pitch, roll, and yaw attitudes with a unit step command are designed. The change of elevator deflection w.r.t. the pitch command of unit step input is shown in Fig. 9. Similarly, the change in ailerons and rudder deflection w.r.t. the roll and yaw command of unit step inputs are shown in Fig. 10 and Fig. 11, respectively.

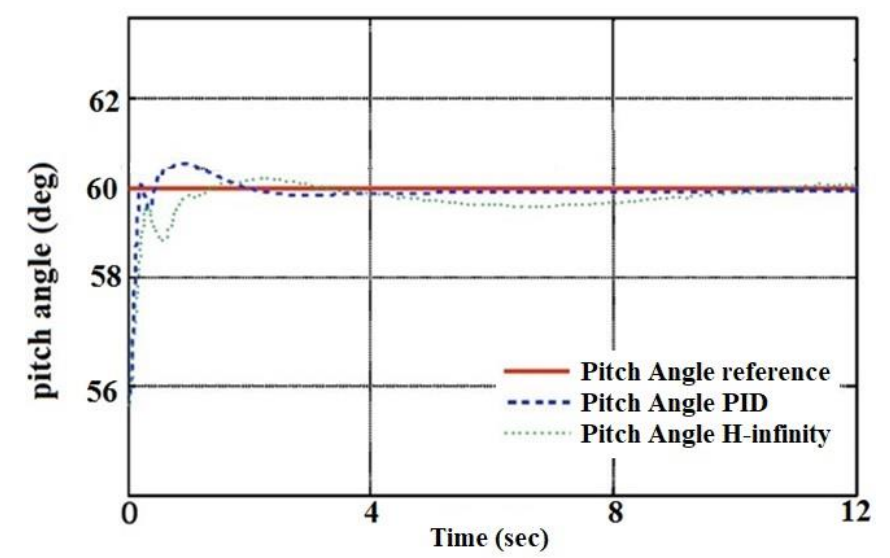

(a)

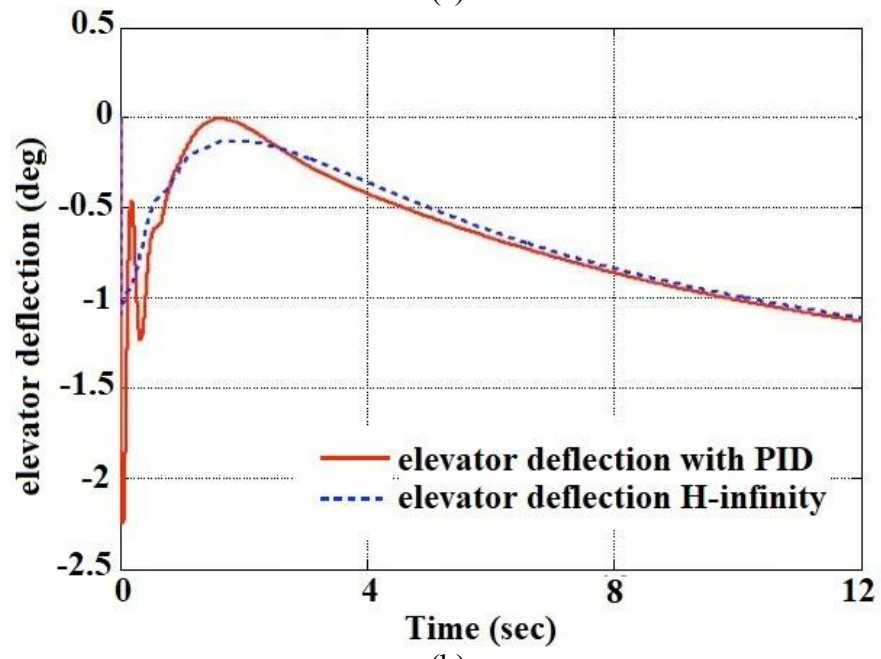

(b)

Fig. 9 (a) Pitch Angle, (b) Elevator deflection. The change in pitch and elevator during entire flight scenario

Long settling time and minimum overshoot are the important parameters in determining the stability of a UAV. The maximum singular value achieved by the sensitivity function $S$ describes the performance of a system. Performance specification indicates that at lower frequencies, the sensitivity function is minimized as low as possible. 


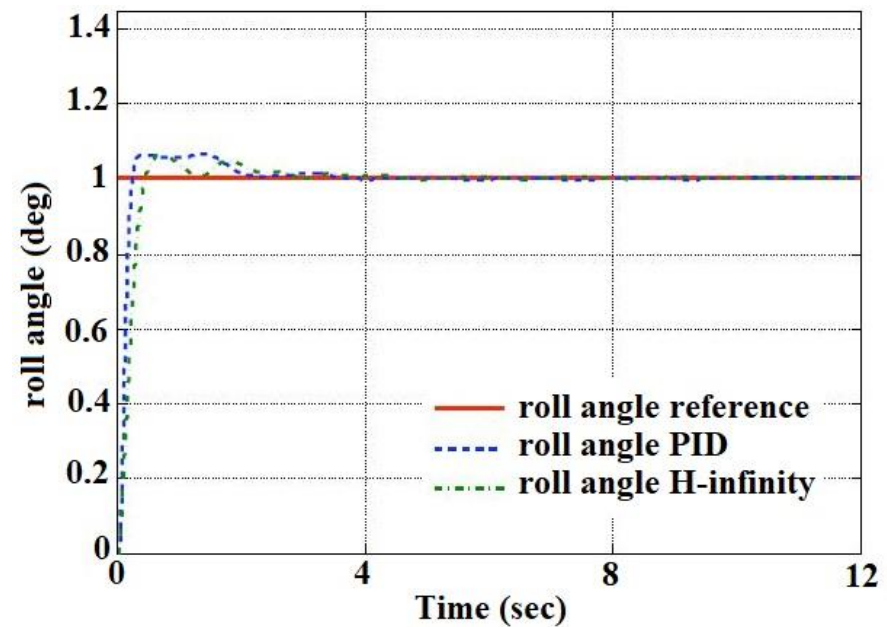

(a)

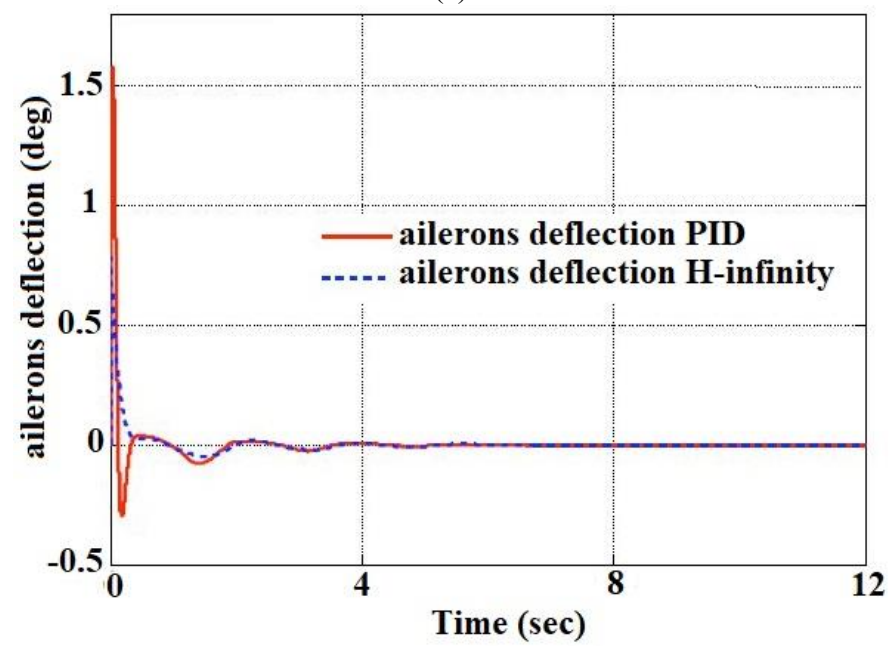

(b)

Fig. 10 (a) Roll Angle, (b) Aileron deflection. The change in roll and aileron during entire flight scenario

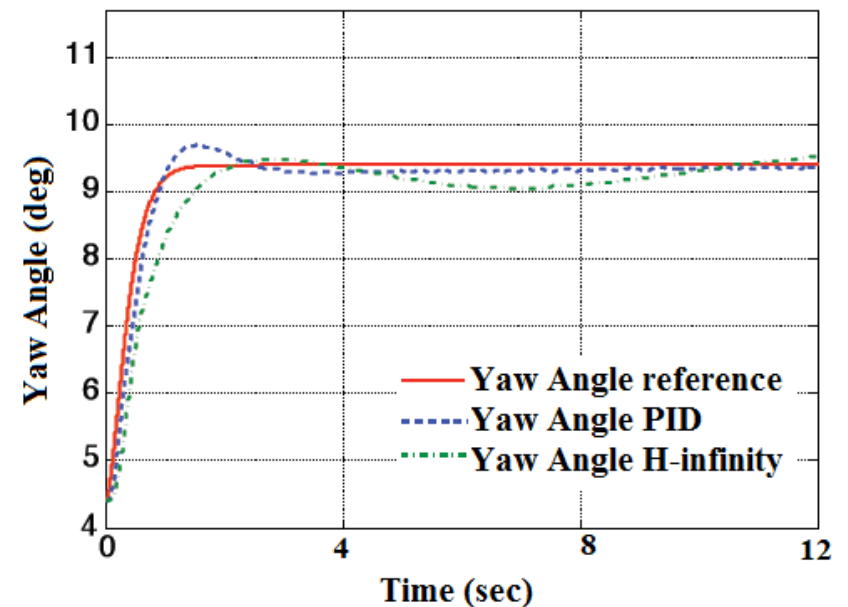

(a)

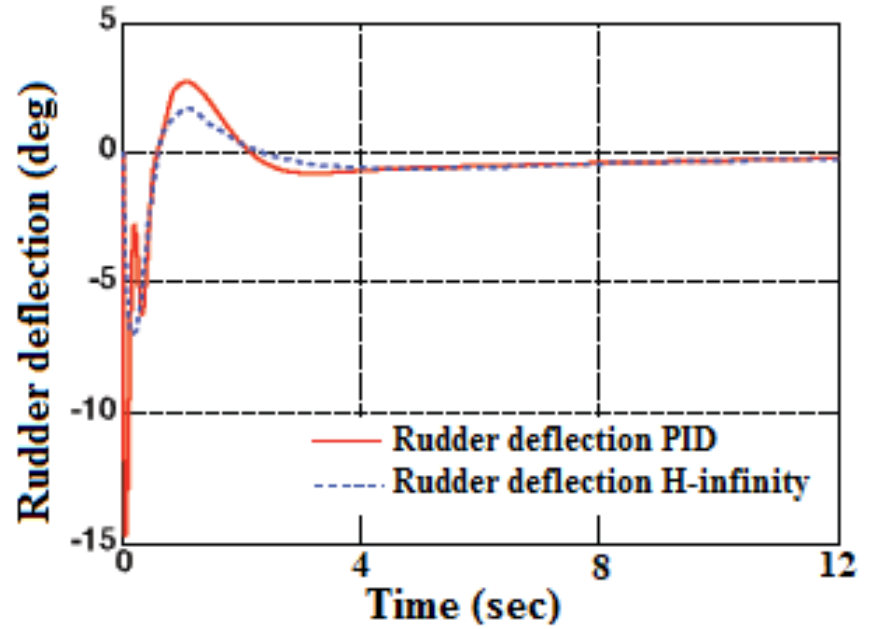

(b)

Fig. 11 (a) Yaw Angle, (b) Rudder deflection. The change in yaw and rudder during entire flight scenario

All the while, a small control effort is maintained at high frequency range. The simulation results of the proposed methods infers that the both PID and robust $\mathrm{H}$-infinity controllers perform excellent in tracking the reference input. In presence of the uncertainty, disturbances and noise, the PID controller is comparatively more sensitive than the robust controller as shown in Fig. 12. Graph infers, due to the presence of random noise and disturbance (air gust), the PID controller has maximum overshoot, and the responses are highly oscillating, and the UAV is missing the trajectory.

From the Fig. 13, the designed PID controller for the UAV without disturbances gives good orientation and position trajectory regulation. In the presence of disturbance (air gust), uncertainty, and random noise, the UAV with PID becomes more unstable having highly oscillatory response and fails to track the trajectory. It is evident from the graphs that minimum overshoot and perfect accuracy in tracking desired trajectory is attained by the designed H-infinity controller. Also, it is more robust against aforementioned conditions by stabilizing the UAV in less time when compared to PID controller.

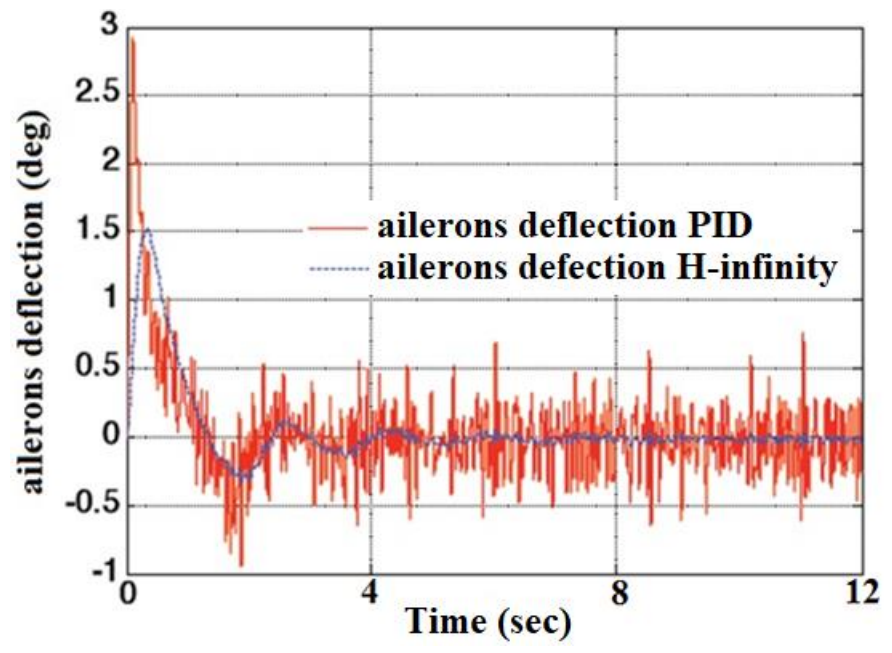

(a) 


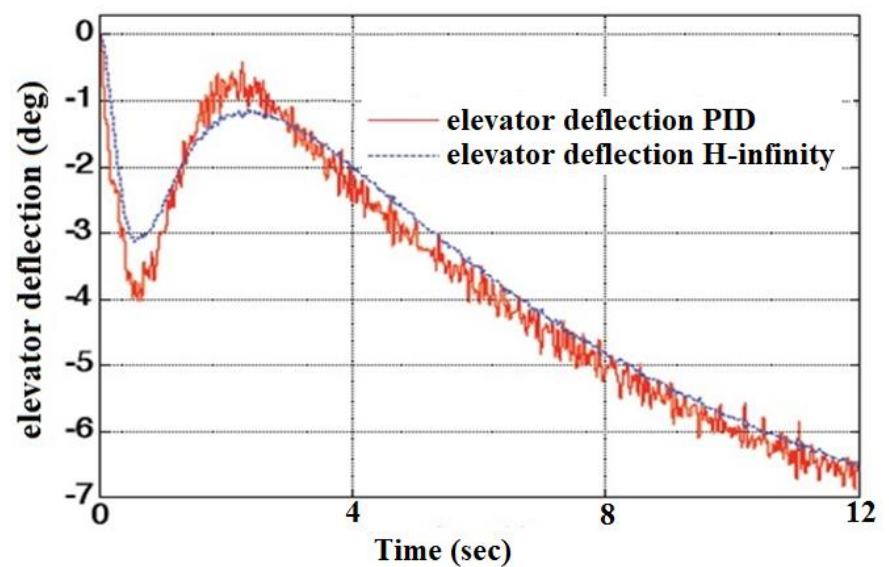

(b)

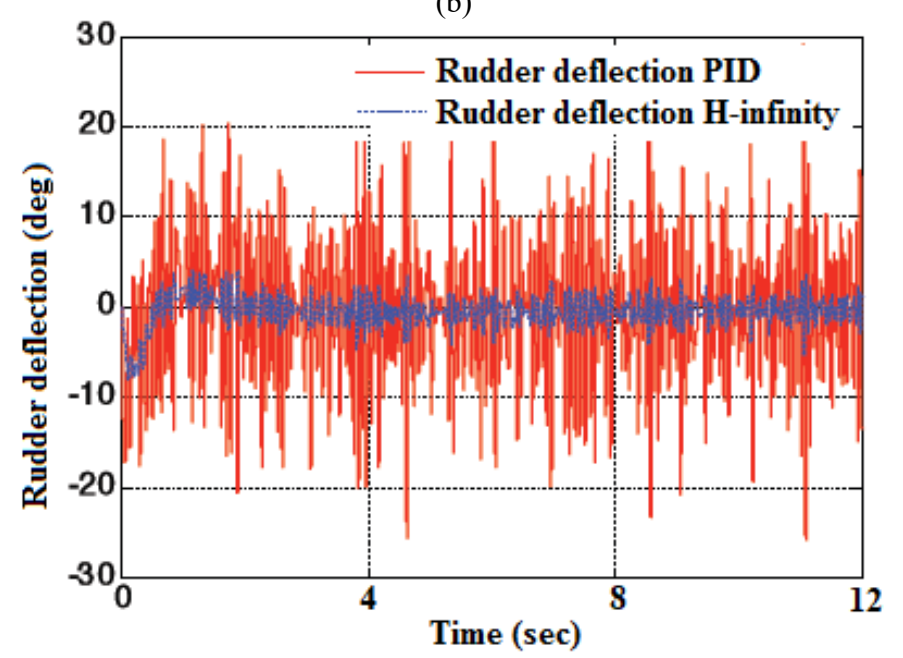

(c)

Fig. 12 (a) Elevator Deflection, (b) Ailerons Deflection, (c) Rudder Deflection. The elevators, ailerons and rudder deflection with noise

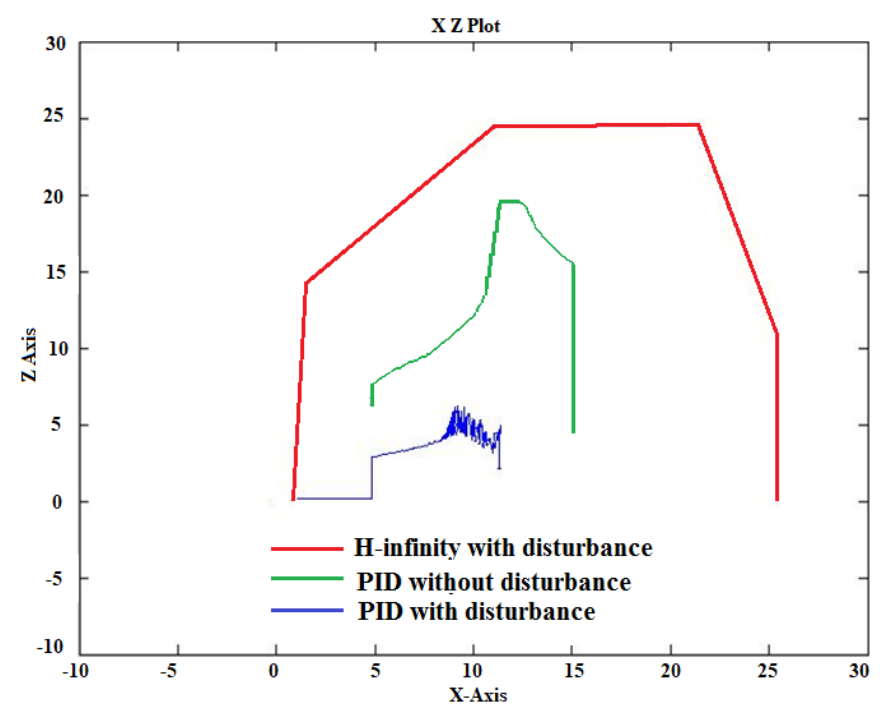

$\mathrm{x}(\mathrm{m})$ coordinate

Fig. 13 The change of flight path with PID and H-infinity controller

\section{A. Robust Performance and Stability Analysis}

By simultaneously shaping the frequency responses for noise reduction, tracking and disturbance rejection, robustness and controller effort, an $\mathrm{H}$-infinity controller is designed. This technique is a useful way to balance the necessary trade-off between performance and robustness as shown in Fig. 14.

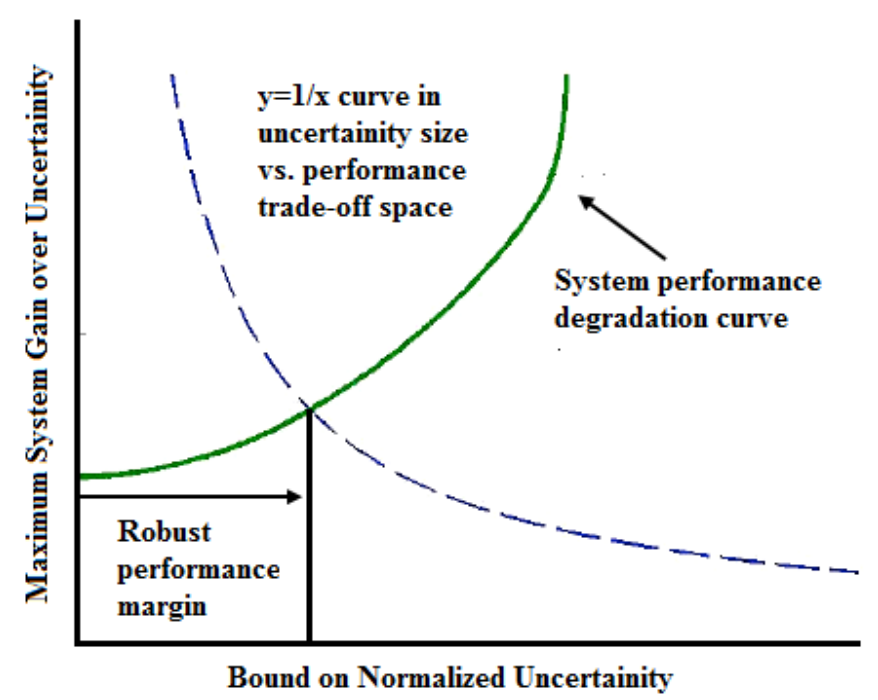

Fig. 14 Tradeoff between performance and uncertainty level

Robust Performance, Robust Stability and Bandwidth: Usually, loop shaping is a tradeoff measure between two contradictory objectives, i.e. robust performance and stability. The robust performance deals with designing $C$, the proper controller which internally stabilizes the feedback system for the given nominal plant. It should also satisfy the inequality $\left\|\left|W_{1} S_{0}\right|+\left|W_{2} T_{0}\right|\right\|_{\infty}<1$. The input data must be Plant $P$, Weighting functions $W_{1}$, and $W_{2}$; and expected output in terms of controller $C$ accomplishing robust performance.

Create $L$, loop transfer function to satisfy ||$\left|W_{1} S_{0}\right|+$ $\left|W_{2} T_{0}\right| \|_{\infty}<1$. Then calculate the controller $C$ using $C=L / P$ [26].

The H-infinity loop shaping procedure can roughly be listed below,

- On the log-log scale, plot two curves with magnitude versus frequency.

- Firstly, plot the graph of $\frac{\left|W_{1}\right|}{\left(1-\left|W_{2}\right|\right)}$ over the low frequency range $\left|W_{1}\right|>1>\left|W_{2}\right|$.

- Secondly, plot the graph of $\frac{\left(1-\left|W_{1}\right|\right)}{\left|W_{2}\right|}$ over the high frequency range $\left|W_{1}\right|<1<\left|W_{2}\right|$.

- $\quad$ Fit the curve of $L$, Loop shaping curve.

- At low frequency, curve $L$ falls above the first curve $\gg 1$.

- At high frequency, curve $L$ falls below the second curve $\ll 1$ and will roll off as fast as $G$ so that the controller $K$ is proper.

- Have a gentle changeover from low to high frequency, maintaining the slope as smooth as possible adjacent to crossover frequency.

- Design a minimum-phase, stable transfer function $L$, so that $L(0)>0$ and the angle of $L$ starts from zero and go on decreasing.

- Robust Stability: At high frequency, the target loop shape gain, $G_{d}$ must be less than $0 d B$. 
- Robust Performance: To achieve good disturbance rejection and high accuracy at low frequency, the Target loop shape gain $G_{d}$ must be more than $0 d B$.

- Crossover: $G_{d}$ crosses the $0 d B$ line between high and low frequency regions called as crossover frequency $W_{c}$.

- Rolloff: $G_{d}$ rolls down with a gradient of -20 to $-40 \mathrm{~dB} /$ decade past the $W_{c}$.

- A target loop shape is calculated using $G_{d}=\frac{w_{c}}{s}$,

where the crossover frequency $w_{c}$ marks the changeover between robust performance and stability.

The responses of plant dynamics are used to decide the target loop shape. Sensitivity, complimentary sensitivity and loop transfer functions are analyzed. In optimal $\mathrm{H}$-infinity loop shaping controller, Sigma as a function of frequency is used to plot the minimum and maximum input-output gains. In this design, $G_{d}(s)$ is $\frac{8}{s}$. The H-infinity controller $K$ matches $G_{d}$ with the open-loop gains $G(s) . K(s)$. The GAM value achieved is 1.4163 which is well within the range of $\pm 3.02 d B$ (using $20 * \log (G A M)=3.02$ ) [4].

Comparison of the plot $G_{d}$ and singular values of $L=G . K$ in the Fig. 15(a) has been carried out. In the Fig. 15(b), the gains of sensitivity function $S$, complementary sensitivity function $T$ and the loop transfer function $L$ are compared. Robust performance and stability range of the designed $\mathrm{H}$ infinity controller is shown in the Fig. 15(c). Therefore, the designed controller $K$ achieves good performance under disturbances and can be used for real time implementations [18].

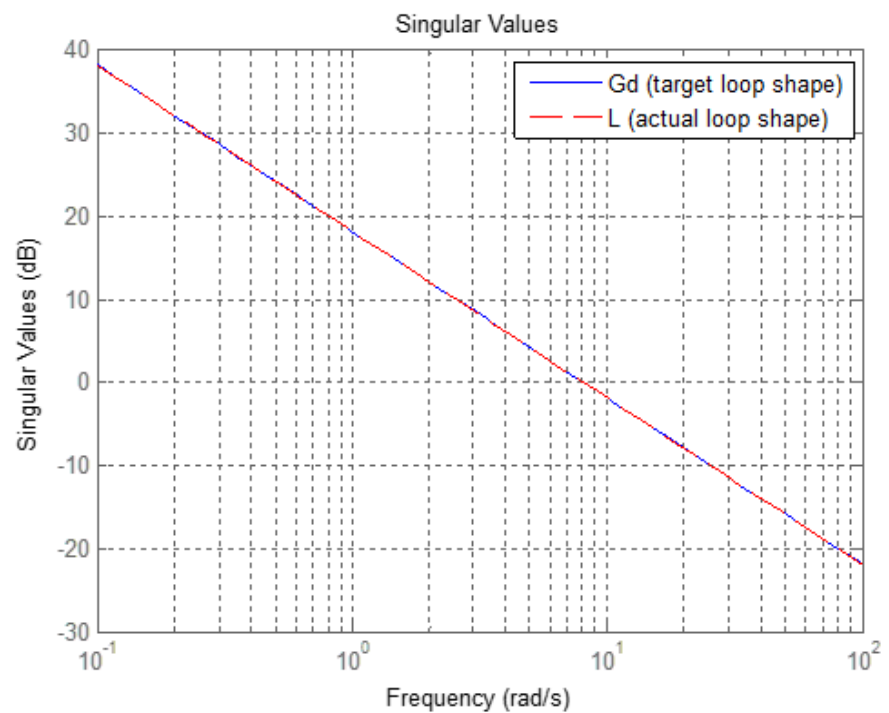

(a)

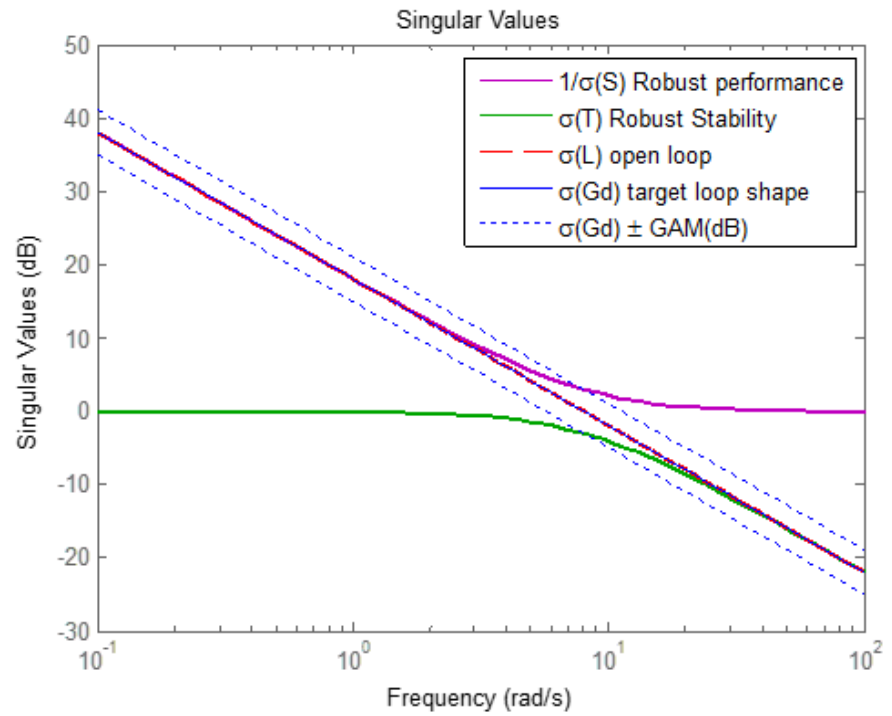

(b)

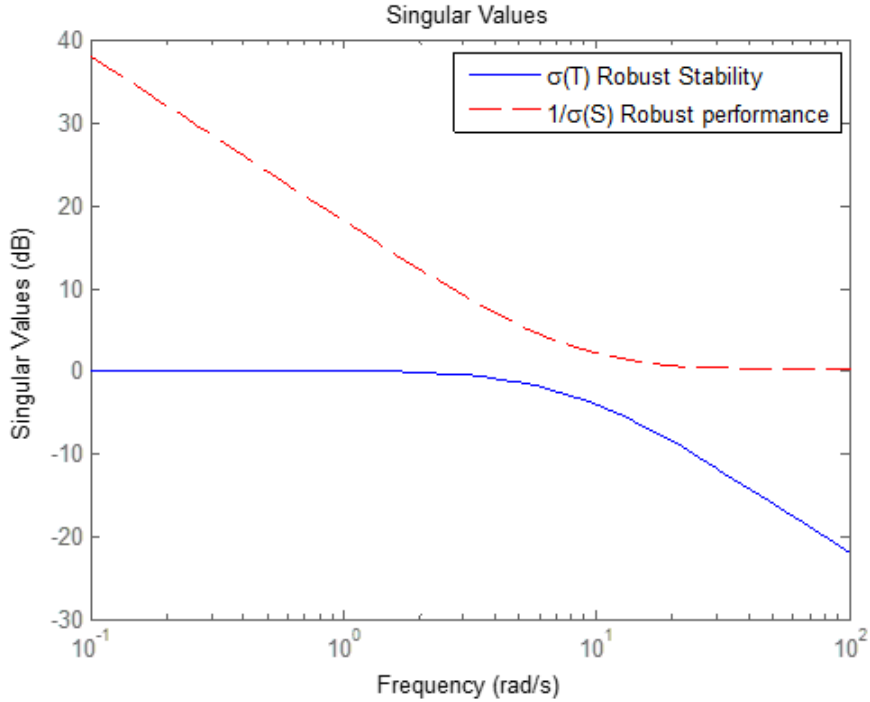

(c)

Fig. 15 (a) Singular values of $L$ compared to $G_{d}$, (b) Sigma frequency response plots, (c) Robust Stability and performance of the UAV

The input/output gain represents the performance of the stable nominal uncertain model, which usually degrades for particular value of its uncertain elements. Furthermore, as these uncertain elements deviate further away from their nominal values, the maximum possible degradation occurs in the performance. The robustperf command provides the necessary information, regarding the robust performance margin in MATLAB, of the uncertain system. Analysis of robust performance basically involves determination of the position, where the dashed-line intersects the degradation curve of system performance, the hyperbola $(y=1 / x)$ as shown in Fig. 14.

The margin of the robust performance is computed through the robustperf command. It provides a measure of the input/output gain degradation. The input/output gain (norm) of the uncertain system will be less than ' 1 ' if and only if the value of the performance margin is greater than ' 1 ' for all values of the uncertainty. The value of the input/output gain is 
greater than ' 1 ' within the particular ranges, only if the performance robustness margin is less than ' 1 ' for certain values of the uncertain elements.

The robustperf instruction presents the necessary information regarding the lower (perfmarg.LowerBound) and the upper (perfmarg.UpperBound) bounds on the performance margin. It also provides the frequency values where the minimal robust performance margin would occur with structure of values of the uncertain elements related to perfmarg.UpperBound and which are associated with upper bound on performance margin. The robustperf command generates another out argument, namely, Frequency vector.

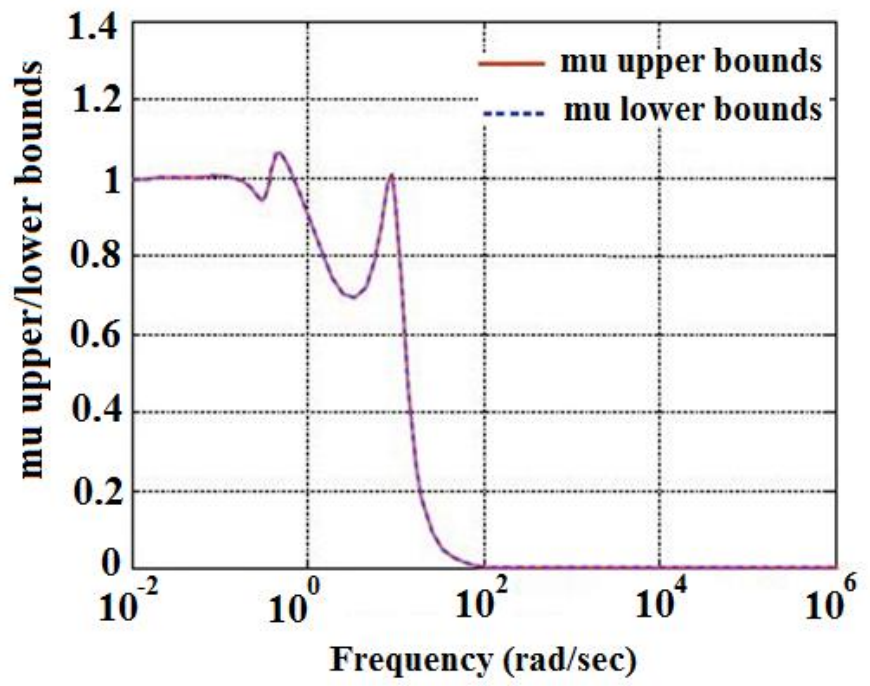

(a)

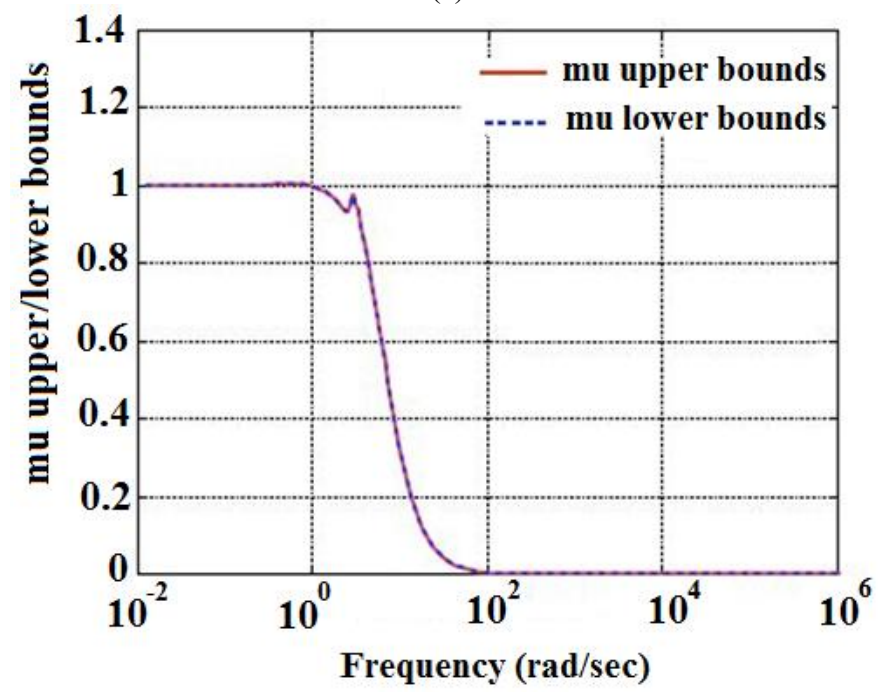

(b)

Fig. 16 (a) Pitch Attitude, (b) Roll Attitude. $\mu$ Bounds for Robust Performance.

This is associated with the Frequency vector (same length as analysis cell array), structure of compressed data and upper and lower bounds from mussv. The mussv argument is useful while computing the lower and upper bounds of the Structured Singular Value $(\mu)$. Uncertain element's structure of values associated with the hyperbola crossing is generated by perfmargunc. To verify that these set of values, they must be substituted into the closed loop system. By following this methodology, it is possible to verify that these values causes the norm of the closed-loop system to be greater than or equal to the reciprocal of the performance margin upper bound: perfmargunc $=1.0567, \quad 1 /($ perfmarg. UpperBound $)=$ 1.0600 as shown in Table I. The Fig. 16 plots the bounds of Structured Singular Value $(\mu)$. The frequency at which the peak occurs is the critical frequency and the performance margin is the reciprocal of the peak value.

Table I. Robust Performance analysis using Lower and Upper Bounds and Critical Frequency

\begin{tabular}{|l|l|l|l|}
\hline Robust Performance & Pitch & Roll & Yaw \\
\hline Lower Bound & 0.934 & 0.907 & 0.912 \\
\hline Upper Bound & 0.934 & 0.907 & 0.912 \\
\hline Critical Frequency & 0.4921 & 0.5460 & 0.6751 \\
\hline
\end{tabular}

The upper and lower bound of the robust performance margin shows that, there is a perturbation about $93 \%$ of uncertainty specified in the sensitivity function with peak gain exceeding 1.5. The stabmarg also gives lower and upper bounds of the robust stability margin, which is a measure of how much uncertainty on controller $K$, the feedback loop can tolerate before becoming unstable. Here the margin is about 0.93, which means that the closed loop will remain stable for up to $93 \%$ of the specified uncertainty.

\section{B. Error Estimation and Comparison using Performance Index}

Performance index is a numerical measure of determining the controller efficiency in terms of integral error w.r.t. reference trajectory. Performance indices are usually categorized into following types: Integral Absolute Error (IAE) in (6), Integral Time-weighted Absolute Error (ITAE) in (7), Integral Squared Error (ISE) in (8), Integral Timeweighted Squared Error (ITSE) in (9) and Mean Square Error (MSE) in (5). The performance of the controller is determined by considering the offset of the achieved output from the setpoint. IAE is the performance index considered for this work. The IAE essentially adds up all the errors as deviation from the setpoint and penalizes the later errors as well. Therefore, reducing the value of IAE would improve the performance of the overall closed-loop system with plant parameters uncertainty. Performances of classical PID and robust $\mathrm{H}$-infinity controller is analyzed using IAE in (6) for pitch attitude controller and shown in Table II.

$$
\begin{aligned}
& I A E=\int|e(t)| d t \\
& I T A E=\int t|e(t)| d t \\
& I S E=\int e^{2} d t \\
& I T S E=\int t e^{2} d t \\
& M S E=\frac{\int e^{2} d t}{T}
\end{aligned}
$$

Error comparison of two different controllers for Quad Tiltrotor UAV with Disturbance, Noise and Uncertainty. 
Table II. Error comparison of two different controllers for Quad Tiltrotor UAV with Disturbance, Noise and Uncertainty

\begin{tabular}{|c|c|c|c|}
\hline $\begin{array}{c}\text { Controller } \\
\text { Method }\end{array}$ & $\begin{array}{c}\text { Pitch } \\
\text { Autopilot }\end{array}$ & $\begin{array}{c}\text { Roll } \\
\text { Autopilot }\end{array}$ & $\begin{array}{c}\text { Yaw } \\
\text { Autopilot }\end{array}$ \\
\hline $\begin{array}{c}\text { Classical } \\
\text { Control }\end{array}$ & 385.2 & 241.7 & 389.8 \\
$\begin{array}{c}\text { Method- } \\
\text { PID }\end{array}$ & & & \\
\hline $\begin{array}{c}\text { Robust } \\
\text { Control } \\
\text { Method- } \\
\text { H-Infinity }\end{array}$ & 94.74 & 213.4 & 173.8 \\
\hline
\end{tabular}

It was illustrated by simulations that both the robust controllers as well as the PID controllers performed similarly well when it comes to tracking reference command for both the nominal plant as well as the plant with aerodynamic uncertainties. Such a behavior is expected from robust controllers since they take the uncertainties of the model into consideration while PID controllers cannot. The fact that even the PID controller was able to handle the uncertainties up to a certain extent, speaks a lot about the reliability of such controllers. The noise considered in this work is at $100 \mathrm{~Hz}$ with a standard deviation of $0.5 \%$. Although both the controllers displayed good tracking performance, the PID controller was observed to be more sensitive to noise. It tends to consider the noise as the input signal and attempts to follow it.

For the VTOL Quad Tiltrotor UAV model obtained through first principles technique, a $\mathrm{H}$-infinity controller which guarantees the robust performance, stability by limiting the peaks of the sensitivity and complementary sensitivity functions, disturbance and noise attenuation is designed. It is not possible to consider all the uncertainties while designing a PID controller, present in the model parameters. The flight path traced by the UAV using the H-infinity controller is accurate when compared to PID with or without disturbances. Simulations are carried out for the nominal model affected by the sensor noise and the uncertainties caused due to variation in aerodynamic derivatives. When the sensor noise is considered, the performance of the designed H-infinity controller surpasses that of the classical PID controller. The errors (IAE) generated by the PID and H-infinity controllers for pitch attitude autopilot are 385.2 and 94.74 , respectively, it can be inferred that the robust $\mathrm{H}$-infinity controller is a good solution for controlling UAV dynamics under the aforementioned conditions.

Based on the graphs obtained, the robust controller has satisfying performance in terms of control surface deflections and Euler angle responses. The PID controller exhibits good reference tracking but is more vulnerable to noise as compared to the robust controller and it exceeds the respective values of the robust controller. Two methodologies are made use of to establish robustness of the robust closed-loop system. The robuststab command describes the basic robustness of the system. $\mu$ analysis is a mathematical tool that can be used to evaluate the robust stability and performance margins. The mussv command calculates the structured singular value, as a dependence of frequency. The results of simulation present the comparison of the controller's performances. Simulations are conducted for all the three conditions, namely, the nominal plant, the plant with aerodynamic uncertainties and the situation where the sensor noise is taken into consideration. In the presence of sensor noise, the performance of the robust controller is superior than that of PID controller. Reducing the derivative time is the most efficient way to decrease the sensitivity of the PID controller. But it also has the downside of increasing the settling time and overshoot. Hence, a compromise in the design of robust $\mathrm{H}$-infinity controller should be made between the system performance and the noise rejection capabilities.

\section{CONCLUSION AND FUTURE WORK}

A linearized 6DoF VTOL Quad Tiltrotor UAV dynamic model with aerodynamics, propulsion, mass inertia and environment models, designed in MATLAB/SIMULINK is considered. The altitude and attitude of a Quad Tiltrotor UAV in the presence of disturbance, uncertainty and noise is controlled by two different techniques. The designed classical PID controller is compared to that of the Robust H-infinity controller. The attitude autopilot performances of both the controllers are compared through simulations and performance index (IAE). The future scope for this work includes the examination of the performance tracking through real time simulation by implementing the model with designed controller into the open-source Flight Gear code.

\section{References}

[1] P. C. Garcia, R. Lozano, and A. E. Dzul, "Modeling and control of miniflying machines," 1st ed. Springer Science \& Business Media, 2005.

[2] M. Miller and J. Narkiewicz, "Tiltrotor Modelling for Simulation in Various Flight Conditions," Journal of Theory and Application Mechanical, vol. 44, no. 4, pp. 881-906, Jan. 2006.

[3] N. T. Hegde, V. I. George, and C. G. Nayak, "Modelling and Transition flight control of Vertical Take-Off and Landing unmanned Tri-Tilting Rotor Aerial Vehicle," in 2019 3rd International conference Electronics Communication. Aerospace Technology Coimbatore, Tamilnadu, India, 2019, pp. 590-594.

[4] N. T. Hegde, V. I. George, C. G. Nayak, and K. Kumar, "Transition flight modeling and robust control of a VTOL unmanned quad tilt-rotor aerial vehicle," Indonesian Journal. Electrical Engineering and Computer Science, vol. 18, no. 3, pp. 1252-1261, 2019.

[5] S. Bouabdallah, A. Noth, and R. Siegwart, "PID vs LQR Control Techniques Applied to an Indoor Micro Quadrotor," in Proceedings 2004 IEEE/RSJ International Conference Intelligent Robots System, Sendal, Japan, 2004, pp. 2451-2456. 
[6] R. A. Hyde, "H-infinity Aerospace Control Design: A VSTOL Flight Application," 1st ed. Springer Science and Business Media, 2005.

[7] S. I. Al Swailem, "Application of Robust Control in Unmanned Vehicle Flight Control System Design," Ph.D. dissertation, College of Aeronautics, Cranfield University, Cranfield, England, 2004.

[8] A. Dharmawan, A. Ashari, and A. E. Putra, "Mathematical modelling of translation and rotation movement in quad tiltrotor," International Journal Advance Science Engineering Information Technology, vol. 7, no. 3, pp. 1104-1113, 2017.

[9] O. Aedo, K. A. Barbosa, and A. Alvarez, "Mixed controller design for an UAV flight control system," in 2015 CHILEAN Conference Electrical Electronics Engineering Information Communication Technology, 2015, pp. 135-140.

[10] G. R. Flores, J. Escareo, R. Lozano, and S. Salazar, "Quad Tilting Rotor Convertible MAV: Modeling and Real-time Hover Flight Control," Journal Intelligent Robot System, vol. 65, no. 1-4, pp. 457-471, Sept. 2011.

[11]C. Uyulan and M. T. Yavuz, "Robust H-infinity control applied on a fixed wing Unmanned Aerial Vehicle," TECHNO-PRESS, 2019.

[12]B. R. Trilaksono, R. Triadhitama, W. Adiprawita, A. Wibowo, and A. Sreenatha, "Hardware-in-the-loop simulation for visual target tracking of octorotor UAV," Aircraft engineering aerospace technology, vol. 83, no. 6, pp. 407-419, Oct. 2011.

[13] A. Mokhtari, A. Benallegue, and B. Daachi, "Robust Feedback Linearization and $G H$-infinity Controller for a Quadrotor Unmanned Aerial Vehicle," Journal of Electrical Engineering, vol. 57, no. 1, pp. 20-27, Apr., 2006.

[14]D. R. Mix, J. S. Koenig, K. M. Linda, O. Cifdaloz, V. L. Wells and A. A. Rodriguez, "Towards Gain-Scheduled Hinfinity Control Design for a Tilt-Wing Aircraft," in 2004 43rd IEEE Conference on Decision Control, Nassau, Bahamas, 2004, pp. 1222-1227.

[15]C. Lin and C. Leng, "Intelligent Robust Control for Uncertain Nonlinear Multivariable Systems via CMAC and H-infinity Technology," in 2008 IEEE International Conference on Systems, Man Cybernetics, Singapore, 2008, pp. 2327-2330.

[16] O. Aedo, K. A. Barbosa, and A. Álvarez, "Mixed controller design for an UAV flight control system," in 2015 CHILEAN Conference on Electrical, Electronics Engineering, Information and Communication Technologies, Santiago, Chile, 2015, pp. 135-140.

[17] A. S. Saeed, A. B. Younes, S. Islam, J. Dias, L. Seneviratne, and G. Cai, "A review on the platform design, dynamic modeling and control of hybrid UAVs," in 2015 International Conference on Unmanned Aircraft Systems, Denver, CO, USA, 2015, pp. 806-815.

[18] N. T. Hegde, V. I. George, C. G. Nayak, and K. Kumar, "Design, dynamic modelling and control of tilt-rotor UAVs: a review," International Journal of Intelligent Unmanned Systems, vol. 8, no. 3, pp. 143-161, Aug., 2019.
[19] A. Prach, "Robust controller design for a fixed wing UAV," Master's Thesis, Dept. of Aerospace Engineering, Middle East Technical University, 2009.

[20]Z. Wang, Z. Gong, Y. Chen, M. Sun, and J. Xu, "Practical control implementation of tri-tiltRotor flying wing unmanned aerial vehicles based upon active disturbance rejection control," Proceedings of the Institution of Mechanical Engineers, Part G: Journal of Aerospace Engineering, vol. 234, no. 4, pp. 943-960, Feb. 2020.

[21]B. Ibrahim and H. Noura, "Formation Flight Control of Multi-UAV System Using Neighbor-based Trajectory Generation Topology," WSEAS Transactions on Applied and Theoretical Mechanics, vol. 15, pp. 173-181, 2021.

[22] N. V. Hien and P. G. Diem, "A Model-Based Design to Implement Controllers for Quadrotor Unmanned Aerial Vehicles," WSEAS Transactions on Systems, vol. 18, pp. 45-61, 2019.

[23] N. T. Hegde, V. I. George, C. G. Nayak, and A. C. Vaz, "Application of robust H-infinity controller in transition flight modeling of autonomous VTOL convertible Quad Tiltrotor UAV," International Journal of Intelligent Unmanned Systems, vol. 9, no. 3, pp.204-235, June 2021.

[24]B. Yuksek, A. Vuruskan, U. Ozdemir, M. A. Yukselen, and G. Inalhan, "Transition Flight Modeling of a FixedWing VTOL UAV," Journal of Intelligent \& Robotic Systems, vol. 84, no. 1-4, pp. 83-105, Jan. 2016.

[25] G.R. Flores and R. Lozano, "Transition Flight Control of the Quad Tilting Rotor Convertible MAV," in 2013 International Conference on Unmanned Aircraft Systems, Atlanta, GA, USA, 2013, pp. 789-794.

[26] K. Zhou and J. C. Doyle, "Essentials of Robust Control," Prentice Hall, 1999.

\section{Creative Commons Attribution License 4.0} (Attribution 4.0 International, CC BY 4.0)

This article is published under the terms of the Creative Commons Attribution License 4.0

https://creativecommons.org/licenses/by/4.0/deed.en_US 